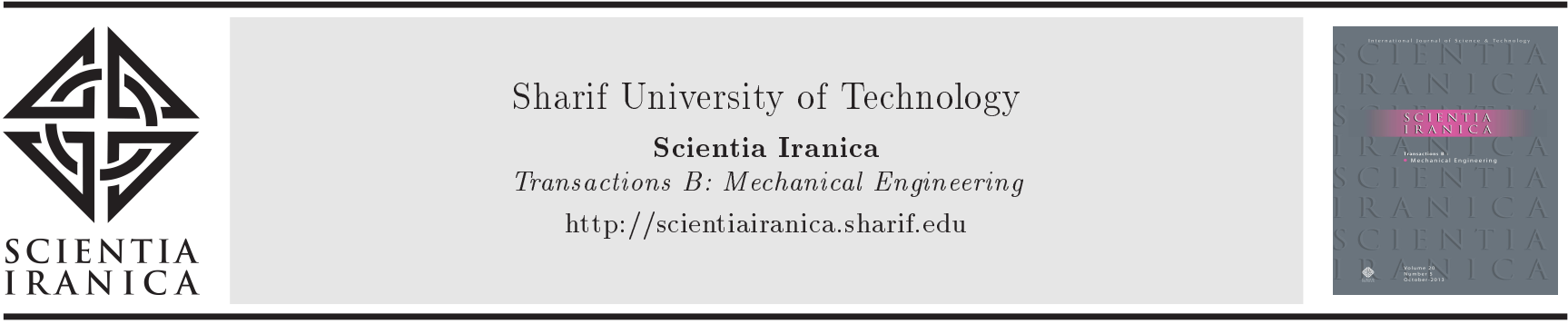

\title{
Hot-film/hot-wire anemometer calibration for low velocities using image processing
}

\author{
M. Behzadi, M.H. Ahmadi, and F. Ommi* \\ Department of Aerospace, Faculty of Mechanical Engineering, Tarbiat Modares University, Tehran, Iran.
}

Received 14 December 2018; received in revised form 23 May 2019; accepted 2 November 2019

\section{KEYWORDS}

Hot-wire anemometer;

Hot-film probe;

Image processing;

Low-speed;

Numerical

differentiation.

\begin{abstract}
Calibration of hot-wire and hot-film probes at low velocities is a difficult task because the dynamic pressure at these velocities is quite low and may not be easily measured. To solve this problem, substituent techniques have been presented in the literature that rely on other phenomena and utilize different hardware. This study described a simple and low-cost method which proposes moving the anemometer probe in the quiescent air (here by means of a swinging arm) and tracked this motion with a camera. After processing the images, the time history of the probe velocity was measured by numerical calculations. Calibration curve was, then, obtained without any predetermined relationship. Using a medium-speed video camera, which is often found in laboratories, would obviate the need for a position sensor and a complicated arm on which this sensor is mounted. This technique can be used for not only pendulums but also other means of moving probes in a quiescent medium.
\end{abstract}

(C) 2021 Sharif University of Technology. All rights reserved.

\section{Introduction}

First introduced several decades ago, constant temperature anemometry is a widely used method among researchers. The probe of these instruments may be either a hot-wire or hot-film. While hot-wires have higher frequency responses making them suitable for measuring rapid changes in velocity like turbulent flows (see [1-3] for instance), hot-films are more robust and sturdier in harsh environments and may also be flush-mounted while measuring wall shear [4]. Probes are found in single- and multi-wire forms to measure one to three velocity components. However, single-

\footnotetext{
*. Corresponding author.

E-mail addresses: milad.behzadi@modares.ac.ir ( $M$. Behzadi); ahmadi.mh@modares.ac.ir (M.H. Ahmadi); fommi@modares.ac.ir (F.Ommi)
}

wire probes are sometimes preferred even for threedimensional fields when the prongs of multi-wire probes disturb the flow $[5,6]$. Calibration of hot-wire and hot-film sensors was performed based on the required velocity range via various methods, varying in terms of availability, cost, and accuracy.

In the range of low velocities, the problem that arise in measuring the dynamic pressure encourages the use of calibration methods not reliant on making a relation between velocity and dynamic pressure. Wind tunnels may be utilized for low-velocity calibration; however, tunnels with two sections of low and high velocity are often required to solve the abovementioned problem, as presented by Pezzotti et al. [7]. A technique was also suggested by Lečić [8] to obtain a large number of calibration points through a single run in wind tunnels which could reduce calibration cost and time. Moreover, a method for investigating the boundary layer on the walls of tunnels was proposed by Grandchamp et al. [9]. The other technique required 
no additional instrument and was based on the dependence of vortex shedding frequency behind cylinders on the flow velocity. Different aspects of this technique may be found in investigations of Ardekani [10], Sattarzadeh et al. [11], and Lee and Budwig [12]. A fully-developed laminar flow in a pipe was also used for calibration, as suggested by Lee and Budwig [12] and Yue and Malmström [13]. In this technique, the flow velocity can be measured according to air volumetric flow rate; however, it may produce an unstable flow which may become an issue [13].

Instead of flowing fluid over the probe, some methods move the probe itself in quiescent fluid. Among the most notable methods include mounting the probe on a rotating arm (see Picatto et al. [14]), on a carriage with linear movement (see Spazzini et al. [15] and Chua et al. [16]), and on a swinging arm (Bruun et al. [17] and Guellouz and Tavoularis [18]). Bruun et al. [17] assumed a known relation between velocity and voltage (King's law) and found its constants by integrating velocities over a fixed probe path. A single equation was considered and ultra-low velocities were ignored. Guellouz and Tavoularis [18] mounted the probe on two radial positions and found the constants by comparing the anemometer outputs for these two locations. Some other calibration methods at low velocities may be found in a review by Özahi et al. [19].

The present study proposes a low-cost method for calibration of hot-wire and hot-film sensors at low velocities and employs a simple cylindrical hotfilm probe. Calibrations for certain applications of hot-films such as measurement of wall shear or other probe shapes are not intended here and can be found elsewhere (see [20-22]). In mounting the probe on a swinging arm, the known technique of moving the probe in quiescent air was utilized. The velocity of the probe was calculated by processing the images captured during its movement. A large number of data points were obtained during a single run. The main difference between this technique and that proposed by Bruun is that in this paper, position is differentiated to give velocity and then, it is compared to Constant Temperature Anemometer (CTA) output to obtain calibration curve. On the contrary, Bruun integrated the CTA output to evaluate the distance and compared it to the distance the probe traveled. Differentiation increases fluctuations of a curve, thus requiring additional processing. However, this technique enjoys two advantages: first, no predetermined calibration equation is needed; second, calibration at a quite low speed is possible given that a separate calibration equation for low velocities may be obtained. The setup and procedure of processing data are presented. Calibration results are then discussed.
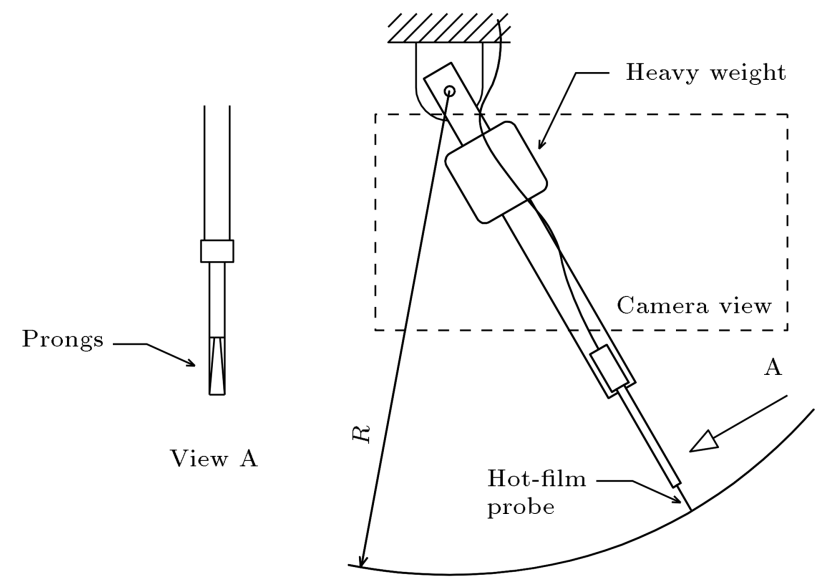

Figure 1. Pendulum and probe orientation.

\section{Calibration setup and procedure}

\subsection{Calibration setup}

The calibration setup includes a constant-temperature anemometer, a data acquisition device, a pendulum that moves the hot-film probe in the air, and a camera to capture images of the arm.

The pendulum comprised a thin, long, and lightweight arm and a heavy-weight as depicted in Figure 1. The weight was attached close to the pivot point to achieve shorter oscillation period and higher velocities. The length of the arm from the pivot point to the probe was $1107 \mathrm{~mm}$. Geometry and mass of the pendulum were kept unchanged in all cases.

The shape of the arm should be carefully determined. In case the shape comprises a large crosssection area, it will induce an airflow affecting the calibration unwantedly; to this end, additional devices or techniques are required to suppress or account for this flow, as presented in [14]. In addition, as the arm passes through the air, it produces turbulence and the vortices left behind are sensed by the probe when the pendulum turns back. This was reported in [18] where only the first swing of the pendulum could have been used for calibration since CTA output indicated strong velocity perturbations in the subsequent swing. However, according to the findings in this paper, no vortex was sensed and the second swing could also be used for calibration. The width of the arm was $3 \mathrm{~mm}$, from the pivot point to $15 \mathrm{~cm}$ left to the probe. The last $15 \mathrm{~cm}$ of the arm was the probe standard support, a rod of $4.6 \mathrm{~mm}$ in diameter. Despite being thin, the arm was rigid enough to preclude lateral vibrations. Vibrations of low frequency can produce an oscillating velocity vector normal to the arm movement plane which cannot be discovered but affect the CTA output.

The anemometer is of constant-temperature type (TSI 1750) and the probe is a cylindrical hot-film type (TSI 1210-20). The ratios of resistances were adjusted to make the probe operate at $250^{\circ} \mathrm{C}$. Data acquisition 
was held using an Advantech 4704 card at a rate of 20 kilo samples per second. This 14-bit device gives a resolution of $2.4 \mathrm{mV}$.

For the anemometer applications, the probe orientation was positioned at an angle from which air flows normally to the plane including the hot-film and the two prongs, as shown in Figure 1. In other words, the flow velocity in the binormal direction and velocities in the normal and parallel directions are zero.

A CASIO EX-F1 camera is placed in front of the pendulum. It was set to capture 600 frames per second. At this speed, image resolution is 432 by 192 . Since the exposure time was short, a $1000 \mathrm{~W}$ lamp was utilized to illuminate the pendulum. After processing, the frame rate was far more than needed. This issue is detailed in the next section.

The experiments were conducted in a closed room. There was no air flow from air conditioning system or doors and windows. Hot-wire and hot-film sensors are generally sensitive to temperature changes. Although there are methods for compensating the temperature changes (see [23]), the instrument used in current experiments was not capable to function similarly. However, this was not regarded as a problem since all experiments were completed in several minutes and the room temperature could not vary at this interval.

\subsection{Calibration procedure}

Probe velocity was calculated based on the angular velocity of the arm. Pendulum motion could not be exactly modeled using a single sine function. Friction and drag make pendulum motion deviate from an ideal sine. Therefore, angular velocity must not be estimated based on the period of oscillation. Moreover, this paper attempts to make use of image processing to find the probe velocity. Therefore, the angle of the arm was extracted from images and the angular velocity was evaluated by differentiating angle with respect to time.

In order to monitor the swinging arm, two redcolored dots were put on the arms $20 \mathrm{~cm}$ apart. These dots could be recognized in the processing of the images throughout the following procedure. Video frames were read into Red/Green/Blue (RGB) format to form three matrices with dimensions the same as those of the image, with each matrix showing the intensity of one color. A parameter (called $I_{r}$ hereafter) was defined for each pixel as the difference between the redness intensity and sum of blueness and greenness intensities. $I_{r}$ had two local maxima in the image area representing the location of the two signs on the arm. The arm tilt could be deduced from the slope of a line passing through these points. This procedure was performed using a computer code prepared for this purpose. Angle estimation error was at most 0.15 degrees in this method. Processing all video frames was not necessary since the frame speed was much more than needed. The time delay between two subsequent processed images was determined to be about $8 \mathrm{~ms}$. In other words, a video record with about $120 \mathrm{fps}$ is adequate for the geometry and range of velocities studied here.

After extracting the angle time histories, the angle was numerically differentiated with respect to time using the second-order central differences. The result was multiplied by the arm length to give velocity. These calculations, done by the computer code, provided velocity time history of the probe. To improve accuracy, another method was also examined which included an extra processing step. It seems that the angle versus frame number curve needs to be smoother. To this end, a three-term Fourier series was fitted to angle frame history using MATLAB software. Of note, a single-term series was not adequate and Fourier series with more three terms would yield the same results. In this respect, to determine velocity, the fitted series, instead of numerical differentiation based on discrete data, were simply derived with respect to the frame number. The improvement achieved through this method will be discussed later.

A camera image may suffer from optical or perspective distortion. One may need to make sure that angles were correctly evaluated from the images. To this end, a circle with equally spaced spokes at known angles was printed on a paper and mounted on a wall in front of the camera. A short video was recorded and then, the angle of spokes in a frame of the video was measured and compared to actual values.

In the last step, CTA output must be synchronized with video frames. In fact, the CTA output is minimum when the pendulum reaches its highest position where the probe velocity becomes zero. This moment was easily found on the angle-frame and voltage-time curves and then, matched. The voltagevelocity curve may be established via the relation between the frame and time. There are sure simpler ways to match videos and CTA output time histories, but this step was first missed and then, after carrying out several tests, this method was determined to be the most efficient one.

Calibration equation fits with the data. Although any type of equation can be used, the simple King's law $E^{2}=A+B V^{n}$ which is a well-known equation in this field is preferred. Here, $E$ is voltage, $V$ is velocity, and $A, B$, and $n$ are constants. Low and very low velocity ranges must be separated since a single equation of this type cannot fit the whole range. Even those who have proposed to modify the King's equation to account for natural convection at near-zero velocities have made this separation [24]. At quite low velocities, constant A was set to $E_{0}$, the CTA voltage at zero velocity. However, for quite higher velocities, this constant was calculated through the fitting process. 
Movement of the arm from vertical position to the highest point and its return was used for the calibration. In order to estimate the uncertainty, it is necessary that the pendulum be released more than once and with different release angles. The variety of release angles made it possible to see whether there were any intrinsic angle-related errors in measuring velocity. This technique would yield accurate and correct results on a condition that the calibration equation be repeatable and not affected by release angle.

\section{Results and discussion}

CTA output is shown in Figure 2 for the three tests performed. The plots are shifted along time axis so that $t=0$ corresponds to the moment at which the pendulum arrives at its highest position. No perturbation is observed, while the response of the anemometer is fast (measured previously to be $35 \mu \mathrm{s}$ ) and the sampling rate is high. This shows that the probe did not face any noticeable disturbance in the air even in return movements.

Probe position was evaluated from video frames. Figure 3 shows the results of the procedure carried out to prove the assumption that the angles can be measured from images. The measured angles of the spokes matched the actual values; in other words, the angles were preserved in the video. The difference between the measured and actual angle values was plotted by a polynomial fitted with it, showing a slight but meaningful trend, as presented in the lower part of Figure 3 . The variability or randomness of error data in this plot was caused by the limitation of pixel density. The error of this type was smaller than 0.15 degrees, as predicted in Section 2.2. However, the fact that the mean trend of errors does not lie on zero indicates that there exists a systematic error in the images. This can be a sign of a very small optical distortion. Here, the accuracy of angle measurement is about 0.1 degrees.

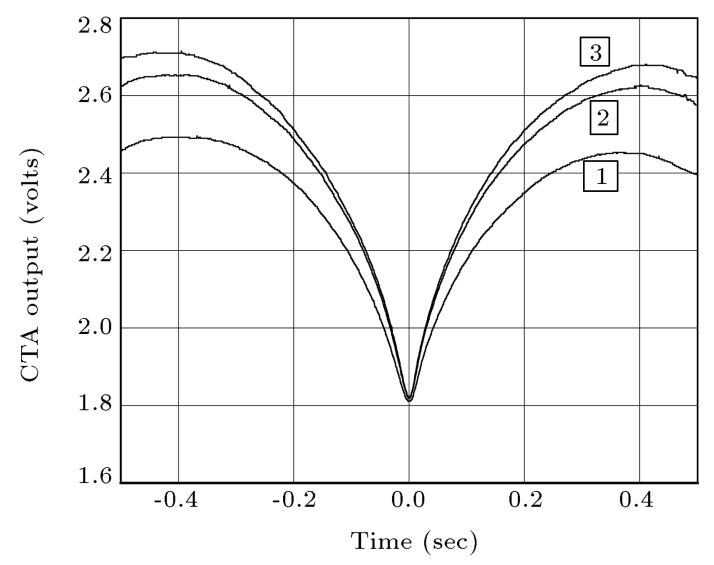

Figure 2. Constant Temperature Anemometer (CTA) output for three releases of the pendulum.

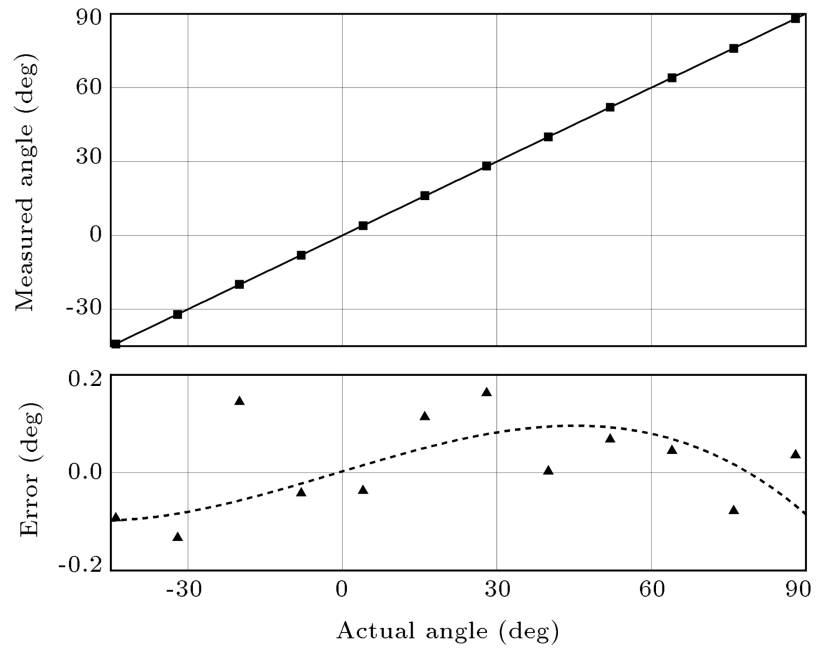

Figure 3. Angle preservation in videos.

In the measurement literature, these two concepts have been defined by precision and accuracy, respectively.

The calibration procedure makes use of a large number of data points and, thus, is not sensitive to random errors. In this respect, the lack of accuracy may not be compensated by increasing the number of data. The current calibration procedure includes the same velocity magnitudes at different angles. Thus, any inaccuracy in angle evaluation would manifest itself as the inconsistency of the final calibration equations and would not remain hidden. However, whenever this technique is to be used, it is a good practice to check the images for possible distortion through a process similar to the current work. Nowadays, cameras usually solve this problem by combining their lenses with internal software. Emergence of distortion in images (optical or perspective) and how it affects calibration may be a subject of study in the future and, if necessary, some known methods of image corrections (e.g. [25]) can be utilized. It is also possible to establish a relation between the real dimensions and the dimensions observed in video recordings.

Figure 4 shows the angle of the arm verses video frame numbers for three releases of the pendulum. Plots are shifted along frame axis similar to anemometer voltage in Figure 2. The solid lines are the result of Fourier series curve fit.

Five moments at which the pendulum came to a stop were captured. These five moments were used to synchronize video frames with CTA output. Figure 5 shows the relation of frame number and time extracted from the data at these five moments along with a line fitted with them. This linear relation was then used for synchronization.

For each of these three releases of the pendulum, two series of voltage-velocity data points were found: one for the forward motion and one for the backward one. As shown in Figure 6, the results of these three 


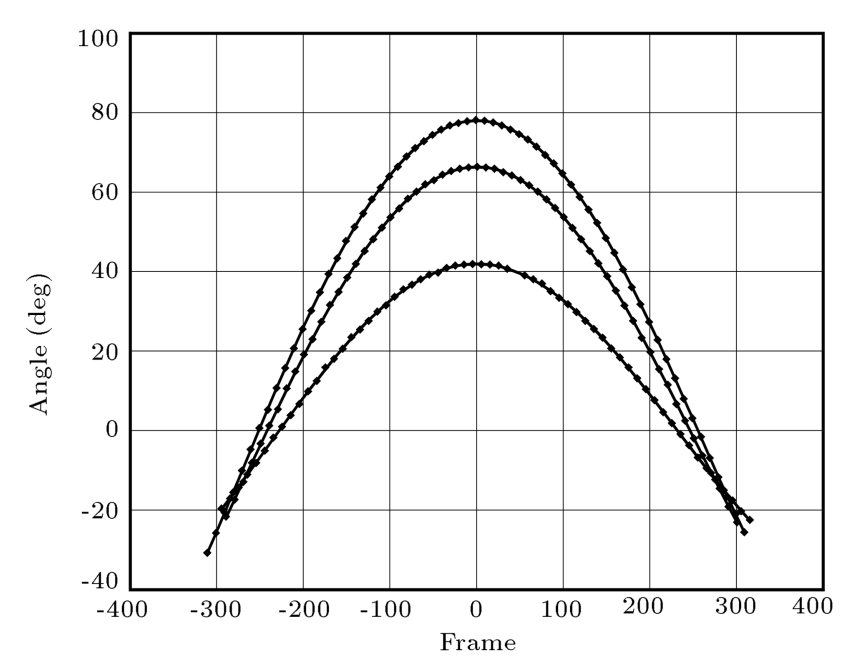

Figure 4. Angle vs. frame obtained through processing of the images for the three releases.

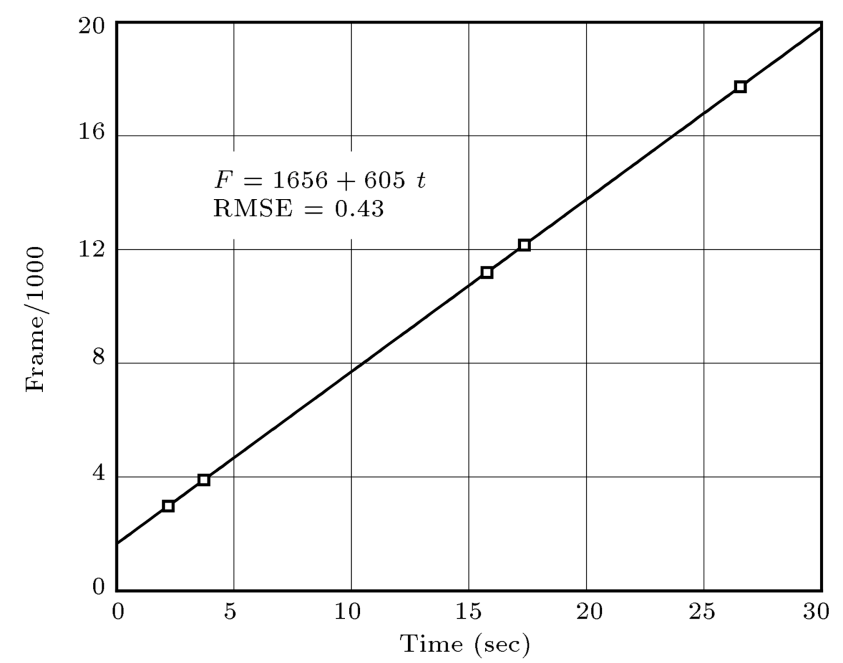

Figure 5. Relation between video frames number and Constant Temperature Anemometer (CTA) output time.

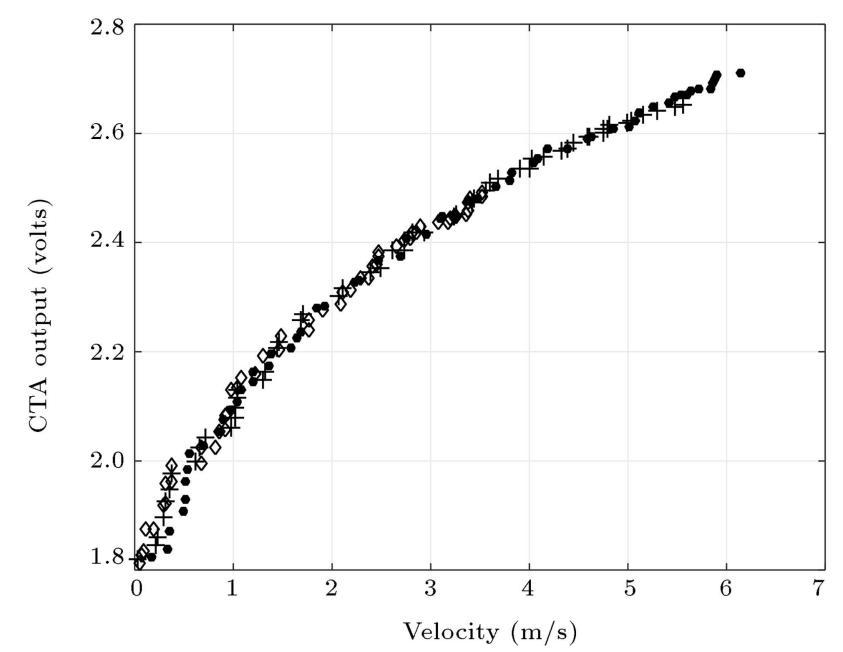

Figure 6. Calibration points from the first (diamonds), second (pluses), and third (circles) releases using direct velocity calculation. tests were in agreement and so were the results of the forward and backward swings of each release; however, this ceased to be true for quite low velocities. The reason might be the free convection around the hot film which was of significance at quite low velocities. When the probe came to stop, the angle of the probe prongs with respect to gravity differed in the tests. In case the two prongs affected the free heat convection around the probe, it was expected that different output voltages would be recorded at the same velocity. Thermal interaction of hot probes and the environment has been the subject of many studies (see [26,27] for instance). If this reasoning is true, it is a drawback to using pendulums and not the technique of using image processing. In this respect, since a single King's law may not be used for the whole range, a calibration equation for velocities greater than $0.6 \mathrm{~m} / \mathrm{s}$ was found. Table 1 presents the calibration constants. Maximum velocity in these experiments is $6.2 \mathrm{~m} / \mathrm{s}$ and the ratio of the upper to lower limits of the range would thus be at most about 10 , which is an acceptable ratio for this type of curve fit.

The uncertainty may be estimated by comparing the three tests. Each test gave a calibration curve which might not exactly coincide with that of the other tests. Using each calibration curve, one can obtain different velocity values for the same CTA output. The third test was assumed the basis of this comparison due to being comprehensive. Test 3 was performed at a higher release angle and thus, contained the whole velocity ranges of Tests 1 and 2 at lower release angles. If Test 1 or 2 was to be chosen as the basis, it would be impossible to compare the whole range of the other tests with it. For each velocity value, the corresponding voltage was evaluated through Test 3 . These voltages were then converted to velocity using calibration curves of the other tests. Relative variations in velocity in this evaluation are shown in Figure 7 . Calibration equation of the test was valid in the velocity range observed. This is why uncertainty of Tests 1 and 2 was evaluated at velocities up to 3.5 and $5.5 \mathrm{~m} / \mathrm{s}$, respectively. The

Table 1. Calibration constants for the three tests.

\begin{tabular}{cccccc}
\hline $\begin{array}{c}\text { Test } \\
\text { number }\end{array}$ & $\begin{array}{c}\text { Velocity } \\
\text { calculation } \\
\text { method }\end{array}$ & $\boldsymbol{A}$ & $\boldsymbol{B}$ & $\boldsymbol{n}$ & $\begin{array}{c}\text { Velocity } \\
\text { range } \\
(\mathbf{m} / \mathbf{s})\end{array}$ \\
\hline 1 & Direct & 1.7321 & 2.6943 & 0.3967 & 3.5 \\
2 & $\begin{array}{c}\text { numerical } \\
\text { differentiation }\end{array}$ & 1.4599 & 2.9447 & 0.3786 & 5.5 \\
3 & & & & & \\
& & 2.0917 & 2.3200 & 0.4521 & 6.2 \\
1 & Using & 2.5294 & 1.9315 & 0.50756 & 3.5 \\
2 & Fourier & 2.6066 & 1.8598 & 0.52127 & 5.5 \\
3 & series & 2.6594 & 1.7922 & 0.53754 & 6.2 \\
\hline
\end{tabular}






Figure 7. Variation in velocities drawn from calibration Tests 1 and 2 relative to Test 3 using direct velocity calculation.



Figure 8. Calibration points from the first (diamonds), second (pluses), and third (circles) releases using Fourier fit.

estimated uncertainty was below $5 \%$ for the whole range and below $1.5 \%$ for the velocities above $1 \mathrm{~m} / \mathrm{s}$.

Calibration curves were also obtained using the second method of velocity calculation based on fitting Fourier series to angle-frame curves. The results are shown in Figure 8. Curves related to the three tests were closer to each other than to those shown in Figure 6. This is more noticeable at lower velocities. Calibration equations of Tests 1 and 2 were compared to that of Test 3 to obtain uncertainty (Figure 9). This time, the uncertainty for the whole range did not exceed $1.5 \%$. Calibration constants of the three tests, shown in Table 1, were also closer to each other. Calibration equations at velocities between 0 and $0.6 \mathrm{~m} / \mathrm{s}$ were evaluated too (not shown here). It was found that in order to have uncertainties better

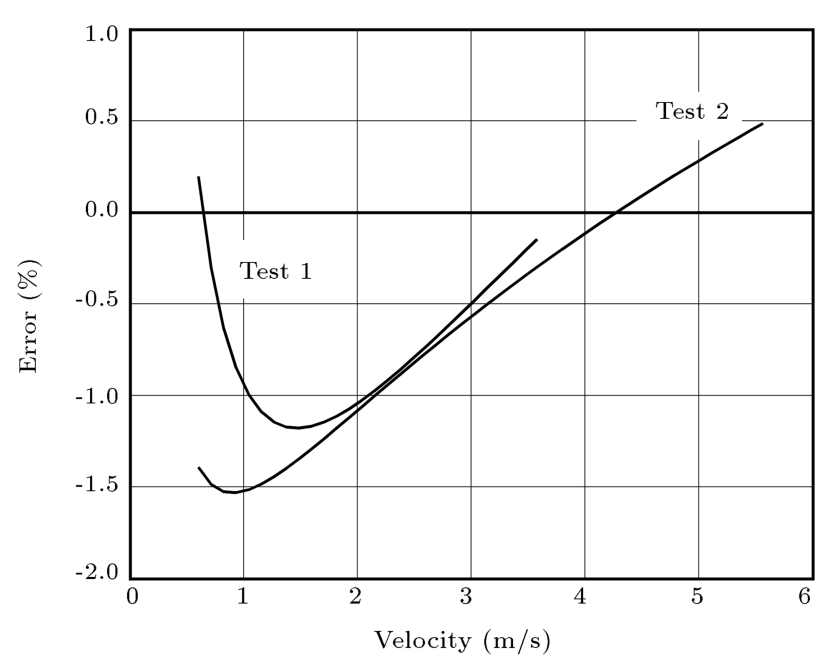

Figure 9. Variation in velocities drawn from calibration Tests 1 and 2 relative to Test 3 using Fourier fit.

than $5 \%$, the range must be limited to velocities higher than $0.1 \mathrm{~m} / \mathrm{s}$. According to these evaluations, application of Fourier series to describe the angle versus frame number history can improve the accuracy of calibration, especially at very low velocities.

\section{Conclusion}

The present study aimed to propose a simple method of calibration of hot-wire and hot-film anemometers at low velocities where methods based on dynamic pressure measurement could not guarantee the required accuracy. This method was applied based on the recorded videos of probe motions in quiescent air. Both the position and velocity of the probe, required for the calibration equation, were determined by processing the video frames. The calibration equation in the range of $0.6-6 \mathrm{~m} / \mathrm{s}$ was obtained and its rate of uncertainty was evaluated. This technique could ensure feasibility and sufficient accuracy. The need for position sensors and complex swing arms was eliminated and a mediumspeed camera was utilized instead, thus making it possible for many researchers to perform calibration with equipment found at their own laboratories. Further findings are listed in the following:

1. If the arm had a small and rigid cross-section, no obtrusive flow or turbulence would be produced. Furthermore, backward swing of the pendulum could be used for calibration;

2. Finding probe velocity by processing captured images provided adequate accuracy for calibration purposes. The minimum required frame rate for the configuration and the velocity range in this study were evaluated;

3. Conformity of calibration curves of different release angles proved that there was no noticeable error 
in velocity estimation that would be dependent on angle since the same velocities occurred at different angles in each release;

4. Describing position time history with Fourier series improved accuracy, especially at very low velocities;

5. It was found that images did not suffer from drastic distortion. However, for future works, the possibility of improving the proposed technique to counteract optical or perspective distortion can be investigated;

6. The main drawback of this technique might be the rather extensive data processing activity that needs a computer code.

\section{References}

1. Davari, A.R., Soltani, M.R., and Ghaeminasab, M. "The unsteady behavior of subsonic wind tunnel wall pressure during pitching motion of the model", Scientia Iranica, 21(1), pp. 192-202 (2014).

2. Davari, A.R., HadiDoolabi, M., Soltani, M.R., Soltani, M.R., and Izadkhah, M. "Aspects of canard-wing vortices interaction in subsonic flow", Scientia Iranica, 22(3), pp. 743-754 (2015).

3. Soltani, M.R., Askari, F., and Sadri, V. "Roughness and turbulence effects on the aerodynamic efficiency of a wind turbine blade section", Scientia Iranica, 23(3), pp. 927-941 (2016).

4. Barratt, D., Nguepnang, M.A., and Atkins, M.D. "Fast-response velocity and shear stress measurements", In Application of Thermo-Fluidic Measurement Techniques: An introduction, Kim T., Lu T.J., and Song S.J, pp. 101-123, Butterworth-Heinemann, Oxford, UK (2016).

5. Berdanier, R.A. and Key, N.L. "A novel data reduction technique for single slanted hot-wire measurements used to study incompressible compressor tip leakage flows", Exp. Fluids, 57(29), pp. 1-4 (2016).

6. Milavec, M., Širok, B., Vidal, D., and Hočevar, M. "Identification of noise generation and flow kinematics in the air gap for two different blade tip designs of an axial fan", Forsch. Ingenieurwes, 79(1), pp. 29-39 (2015).

7. Pezzotti, S., D'Iorio, J.I., Nadal-Mora, V., and Pesarini, A. "A wind tunnel for anemometer calibration in the range of $0.2-1.25 \mathrm{~m} / \mathrm{s}$ ", Flow Meas. Instrum., 22(4), pp. 338-342 (2011).

8. Lečić, M.R. "A new experimental approach to the calibration of hot-wire probes", Flow Meas. Instrum., 20(3), pp. 136-140 (2009).

9. Grandchamp, X., Van Hirtum, A., and Pelorson, X. "Hot film/wire calibration for low to moderate flow velocities", Meas. Sci. Technol., 21(11), p. 115402 (2010).

10. Ardekani, M.A. "Hot-wire calibration using vortex shedding", Measurement, 42(5), pp. 722-729 (2009).
11. Sattarzadeh, S.S., Kalpakli, A., and Örlü, R. "Hotwire calibration at low velocities: Revisiting the vortex shedding method", Adv. Mech. Eng., p. 241726 (2013).

12. Lee, T. and Budwig, R. "Two improved methods for low-speed hot-wire calibration", Meas. Sci. Technol., 2(7), pp. 643-646 (1991).

13. Yue, Z. and Malmström, G. "A simple method for lowspeed hot-wire anemometer calibration", Meas. Sci. Technol., 9(9), pp. 1506-1510 (1998).

14. Piccato, A., Malvano, R., and Spazzini, P.G. "Metrological features of the rotating low-speed anemometer calibration facility at INRIM", Metrologia, 47(1), pp. 47-57 (2010).

15. Spazzini, P.G., Piccato, A., and Malvano, R. "Metrological features of the linear low-speed anemometer calibration facility at INRIM", Metrologia, 46(1), pp. 109-118 (2009).

16. Chua, L.P., Li, H.-S., and Zhang, H. "Calibration of hot wire for low speed measurements", Int. Commun. Heat Mass Transf., 27(4), pp. 507-516 (2000).

17. Bruun, H.H., Farrar, B., and Watson, I. "A swinging arm calibration method for low velocity hot-wire probe calibration", Exp. Fluids, 7(6), pp. 400-404 (1989).

18. Guellouz, M.S. and Tavoularis, S. "A simple pendulum technique for the calibration of hot-wire anemometers over low-velocity ranges", Exp. Fluids, 18(3), pp. 199203 (1995).

19. Özahi E., Çarpınlığlu, M.Ö., and Gündoğdu, M.Y. "Simple methods for low speed calibration of hot-wire anemometers", Flow Meas. Instrum., 21(2), pp. 166170 (2010).

20. Krause, M., Gaisbauer, U., Kraemer, E., and Kosinov, A.D. "Implementation of a new thermal model and static calibration of a wedge-shaped hot-film probe in a constant-temperature mode", Int. J. Heat Mass Transf., 126(A), pp. 1-9 (2018).

21. Liu, X., Li, Z., and Gao, N. "An improved wall shear stress measurement technique using sandwiched hotfilm sensors", Theor. Appl. Mech. Lett., 8(2), pp. 137141 (2018).

22. Kaiser, E. "Increase factor for forced convection at small heating elements", Forsch. Ingenieurwes., 82(1), pp. 9-20 (2018), (in German).

23. Miheev, N.I., Molochnikov, V.M., Kratirov, D.V., Hayrnasov, K.R., and Zanko, P.S. "Hot-wire measurements with automatic compensation of ambient temperature changes", Therm. Sci., 19(2), pp. 509$520(2015)$.

24. Johansson, A.V. and Alfredsson, P.H. "On the structure of turbulent channel flow", J. Fluid Mech., 122(1), pp. 295-314 (1982).

25. Moravec, J. and Hub, M. "Automatic correction of barrel distorted images using a cascaded evolutionary estimator", Inform. Sciences, 366(1), pp. 70-98 (2016). 
26. Ikeya, Y., Örlü, R., Fukagata, K., and Alfredsson, P.H. "Towards a theoretical model of heat transfer for hotwire anemometry close to solid walls", Int. J. Heat Fluid Flow, 68(1), pp. 248-256 (2017).

27. Sun, B., Ma, B., Luo, J., Li, B., Jiang, C., and Deng, J. "Sensing elements space design of hot-film sensor array considering thermal crosstalk", Sensor. Actuat. A-Phys., 265(1), pp. 217-223 (2017).

\section{Biographies}

Milad Behzadi is presently a $\mathrm{PhD}$ Candidate at Tarbiat Modares University, Tehran Iran. He completed his MS in Aerospace Engineering in the field of propulsion in 2012 at Sharif University of Technology and obtained his BE in Mechanical Engineering from Shahid Bahonar University, Iran, as the top student. He has guided some graduate students and his research works include design and application of measuring instruments, combustion instability, and optical measurements in fluids.

Mohammad Hasan Ahmadi, born in 1992, earned his MS degree in Aerospace Engineering at Tarbiat Modares University and his BE in Mechanical Engineering from Hamedan University, Iran. His research interests are industrial combustion, flow turbulence, and gas dynamics.

Fatholah Ommi is the President of Aerospace Research Institute of the Islamic Republic of Iran and also a Professor at Tarbiat Modares University, Iran. He obtained his PhD degree in Mechanical Engineering from Moscow Technological University, Russia in 1996. He has been the author of more than a hundred scientific papers and three text books. Propulsion systems, fuel cells, and spray modeling are among his research interests. 